\title{
Rescuing an Entrapped Guidewire Using a Tornus ${ }^{\circledR}$ Catheter
}

\author{
Yun-Hyeong Cho, MD; Sungha Park, MD, PhD*; Jung-Sun Kim, MD*; \\ Seung-Yul Lee, MD*; Donghoon Choi, MD, PhD*; \\ Yangsoo Jang, $\mathrm{MD}, \mathrm{PhD}^{*}$; Seung-Yun Cho, $\mathrm{MD}, \mathrm{PhD}^{*}$
}

\begin{abstract}
Entrapment of a guidewire is a rare event, but it could happen especially in severe calcified stenotic lesion. This complication is life-threatening because it can lead to myocardial ischemia, infarction, or lethal arrhythmia due to intracoronary thrombosis. In the event of failed retrieval and persistent signs of ischemia, patients should be urgently referred to surgery. During percutaneous coronary intervention, the Tornus catheter has been applied to patients with severe coronary artery disease when balloon catheter or microcatheter cannot be crossed across the lesion after successful wire crossing. This is a case of a patient that a Tornus catheter rescued an entrapped guidewire in severe calcified coronary disease. (Circ J 2007; 71: 1326-1327)
\end{abstract}

Key Words: Angioplasty; Calcium; Complications

$\mathbf{T}$ he Tornus ${ }^{\circledR}$ (Asahi Intecc, Aichi, Japan) catheter (Fig 1) has been developed as a novel over-the-wire catheter for severe coronary artery disease (CAD). During percutaneous coronary intervention (PCI), it is used in patients with severe CAD when a balloon catheter or microcatheter cannot be passed across the lesion after successful gudewire crossing! Entrapment of a guidewire or an intact angioplasty balloon is a rare event, but can happen, especially in a severely calcified stenotic lesion. This is a case of a patient in wom a Tornus catheter was used to successfully rescue a guidewire entrapped in severely calcified coronary disease.

\section{Case Report}

A 75-year-old woman with a history of diabetes for 20 years and hypertension for 3 years came to the emergency room complaining of acute onset of dyspnea. On arrival, surface ECG demonstrated significant depression of ST segments in the $\mathrm{V}_{4-6}$ precordial leads, and there was elevation of troponin T and CK-MB cardiac enzymes. Her blood pressure was $96 / 50 \mathrm{mmHg}$ at the time of admission.

Emergency coronary angiography was performed on the next day of admission because she developed hemodynamic instability. Coronary angiography was attempted via the right femoral artery, and intra-aortic balloon counterpulsation was performed via the left femoral artery. After the placement of an 8Fr femoral sheath, coronary angiography was carried out using a 5Fr Judkin left and right catheter. Angiography revealed chronic total occlusion of the midportion of the left anterior descending artery, significant stenosis of the distal left circumflex artery with heavy calci-

(Received March 5, 2007; revised manuscript received April 13, 2007; accepted April 24, 2007)

Divisions of Cardiology, Kwandong University College of Medicine, Goyang, *Yonsei University College of Medicine, Seoul, Korea Mailing address: Donghoon Choi, MD, PhD, Cardiology Division, Yonsei University College of Medicine, Yonsei Cardiovascular Center, CPO Box 8044, Seoul 120-752, South Korea. E-mail: cdhlyj@ yumc.yonsei.ac.kr fication (Fig 2A), and diffuse irregular severe stenosis of the right coronary artery. It was decided to perform percutaneous transluminal coronary angioplasty of the distal left circumflex artery. After the placement of a 7Fr EBU guiding catheter, a 0.014-inch soft guidewire (Asahi, Japan) was passed through the stenotic lesion of the distal circumflex artery. Next, a $1.5 \times 15 \mathrm{~mm}$ Sprinter balloon (Medtronic, USA) was used to pass the lesion, but failed, so a $1.25 \times$ $15 \mathrm{~mm}$ Ryujin balloon (Terumo, Japan) was tried, but failed as well (Fig 2B). Because of the failure to pass an angioplasty balloon across the lesion, emergency coronary artery bypass surgery was planned. However, the guidewire could not be retrieved because it was entrapped in the distal circumflex artery, most likely because of the severe calcification. We tried to remove the guidewire by several methods, such as gentle manipulation of the guidewire, using another guidewire, and ballooning of the proximal lesion with a $1.25 \times 15 \mathrm{~mm}$ Ryujin balloon several times, but nothing was helpful. Finally, we decided to use a 2.1Fr Tornus catheter to advance through the most calcified lesion (Fig 2C) and after its passage through the lesion, we were able to retrieve

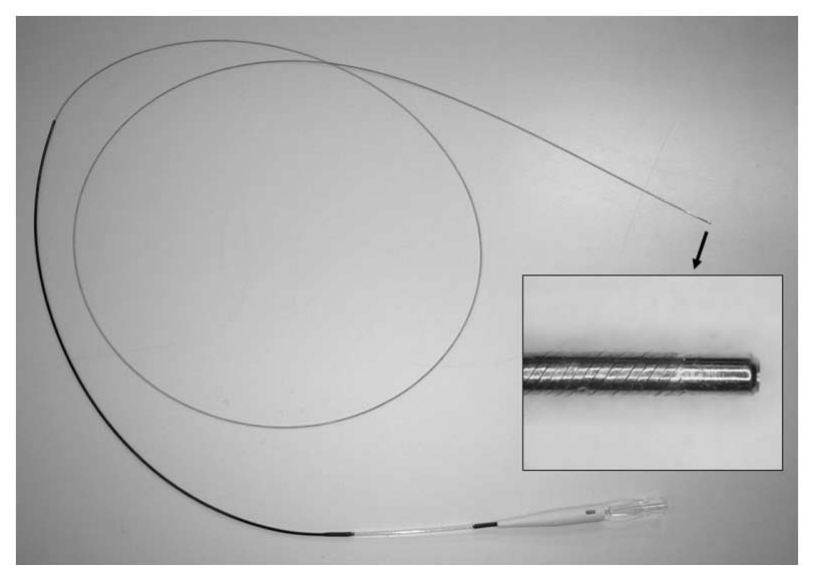

Fig 1. Tornus catheter. 


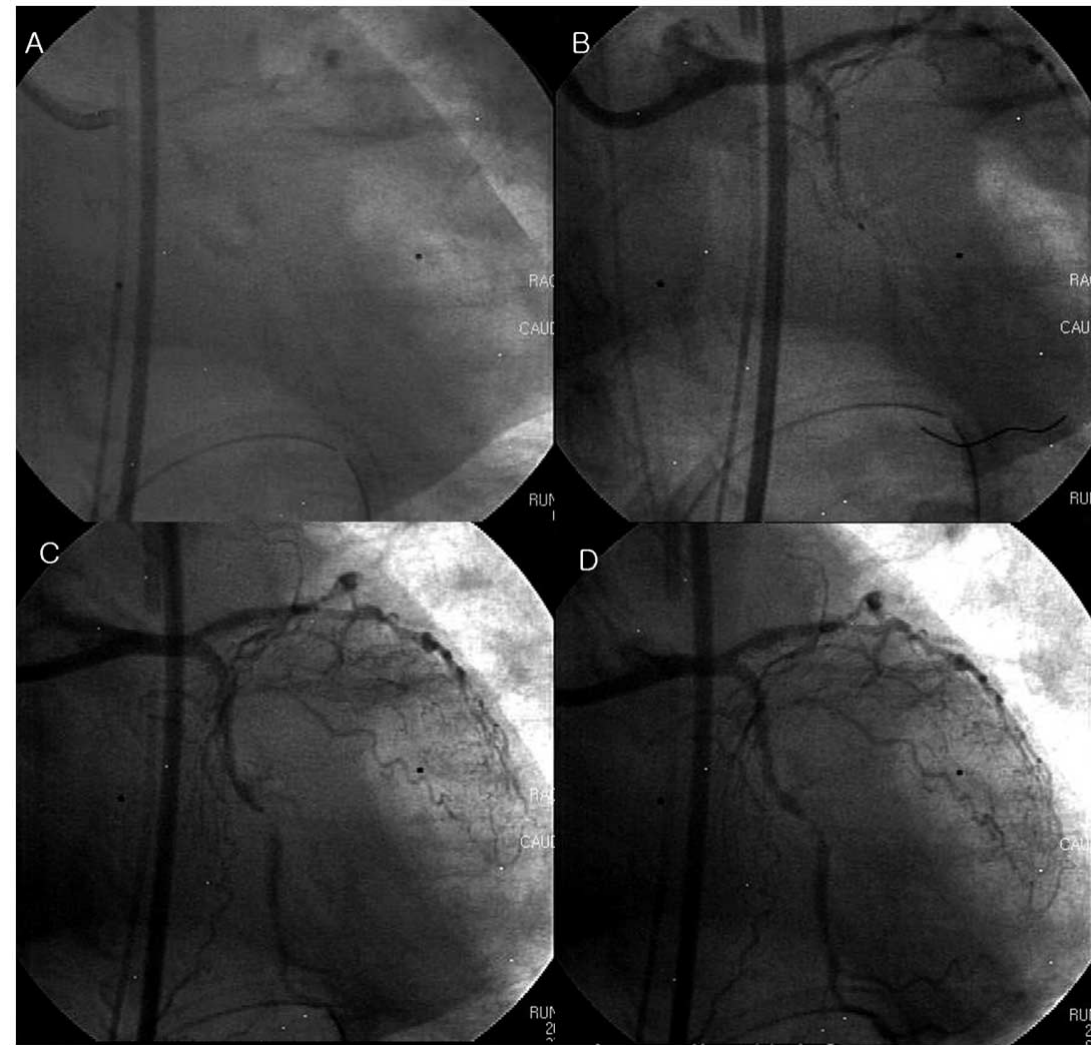

Fig 2. (A-D) Successful removal of the guidewire entrapped in the severely calcified lesion using the Tornus catheter. the guidewire (Fig 2D). Meanwhile, we had already used a large amount of contrast, and because it was not certain that the Tornus catheter would be able to completely cross the calcified lesion, we decided to refer the patient for emergency coronary artery bypass surgery, which was successful.

\section{Discussion}

Entrapment of a guidewire in a coronary artery is a rare but serious, life-threatening complication of PCI. It is lifethreatening because it can lead to myocardial ischemia, infarction, or lethal arrhythmia because of intracoronary thrombosis. In the event of failed retrieval and persistent signs of ischemia, the patient requires urgent surgery?

In the past few years many devices have been introduced to give the operator and patient a greater chance of successful revascularization of a chronic total occlusion (CTO). Even when a guidewire is successfully passed through the lesion, severe coronary artery stenosis, such as severe calcification or CTO, can prevent the passing of a conventional balloon or a microcatheter. Ablation with high-speed rotational atherectomy is efficacious for these lesions, but requires exchange of a guidewire for a RotaWire (Boston Scientific, Miami, FL) before ablation, and the exchange procedure is not always successful when there is not a catheter passing through the lesion.
The Tornus catheter acts as a support catheter and was designed to be used in situations where support is imperative. It can pass through severe stenosis easily with counterclockwise rotation along the guidewire because the shaft is stranded clockwise! It is especially useful when the guidewire is entrapped in severly calcified stenotic lesion because it does not need to exchange the guidewire. In the present case, the Tornus catheter, a novel penetrating catheter, was used to retrieve an entrapped guidewire in a severely calcified coronary lesion.

\section{References}

1. Tsuchikane E, Katoh O, Shimogami M, Ito T, Ehara M, Sato H, et al. First clinical experience of a novel penetration catheter for patients with severe coronary artery stenosis. Cathet Cardiovasc Intervent 2005; 65: $368-373$.

2. Baim DS, Braden G, Heuser R, Popma JJ, Cutlip DE, Massaro JM, et al; Guided Radio Frequency Energy Ablation of Total Occlusions Registry Study. Utility of the Safe-Cross-guided radiofrequency total occlusion crossing system in chronic coronary total occlusions (results from the Guided Radio Frequency Energy Ablation of Total Occlusions Registry Study). Am J Cardiol 2004; 94: 853-858.

3. Alexiou K, Kappert U, Knaut M, Matschke K, Malte Tugtekin S. Entrapped coronary catheter remnants and stents: Must they be surgically removed? Tex Heart Inst J 2006; 33: 139-142.

4. Henneke KH, Regar E, Konig A, Werner F, Klauss V, Metz J, et al. Impact of target lesion calcification on coronary stent expansion after rotational atherectomy. Am Heart J 1999; 137: 93-99. 